\title{
Weighted Morrey Estimates for Multilinear Fourier Multiplier Operators
}

\author{
Songbai Wang, Yinsheng Jiang, and Peng Li \\ College of Mathematics and System Sciences, Xinjiang University, Urumqi 830046, China \\ Correspondence should be addressed to Yinsheng Jiang; ysjiang@xju.edu.cn
}

Received 7 December 2013; Revised 10 February 2014; Accepted 13 February 2014; Published 31 March 2014

Academic Editor: Dashan Fan

Copyright (C) 2014 Songbai Wang et al. This is an open access article distributed under the Creative Commons Attribution License, which permits unrestricted use, distribution, and reproduction in any medium, provided the original work is properly cited.

The multilinear Fourier multipliers and their commutators with Sobolev regularity are studied. The purpose of this paper is to establish that these operators are bounded on certain product Morrey spaces $L^{p, k}\left(\mathbb{R}^{n}\right)$. Based on the boundedness of these operators from $L^{p_{1}}\left(\omega_{1}\right) \times \cdots \times L^{p_{m}}\left(\omega_{m}\right)$ to $L^{p}\left(\prod_{j=1}^{m} \omega^{p / p_{j}}\right)$, we obtained that they are also bounded from $L^{p_{1}, k}\left(\omega_{1}\right) \times \cdots \times L^{p_{m}, k}\left(\omega_{m}\right)$ to $L^{p, k}\left(\prod_{j=1}^{m} \omega^{p / p_{j}}\right)$, with $0<k<1,1<p_{j}<\infty, 1 / p=1 / p_{1}+\cdots+1 / p_{m}$, and $\omega_{j} \in A_{p_{j}}, j=1, \ldots, m$.

\section{Introduction}

Recently some authors have taken so much interest in the text of multilinear Fourier multipliers with Sobolev regularity. To state some interesting results, we recall some necessary notations and definitions. Let $\sigma \in L^{\infty}\left(\mathbb{R}^{m n}\right)$; the multilinear Fourier multiplier operator $T_{\sigma}$ is defined by

$$
\begin{aligned}
T_{\sigma}(\vec{f})(x)=\int_{\mathbb{R}^{m n}} & \exp \left(2 \pi i x\left(\xi_{1}+\cdots+\xi_{m}\right)\right) \\
& \times \sigma\left(\xi_{1}, \ldots, \xi_{m}\right) \widehat{f}_{1}\left(\xi_{1}\right) \cdots \widehat{f}_{m}\left(\xi_{m}\right) d \vec{\xi}
\end{aligned}
$$

for all $\vec{f}=\left(f_{1}, \ldots, f_{m}\right) \in \mathcal{S}\left(\mathbb{R}^{n}\right)^{m}$, where $d \vec{\xi}=d \xi_{1} \cdots d \xi_{m}$ and $\widehat{f}$ is the Fourier transform of $f$. It is well known that [1] the boundedness of $T_{m}$ from $L^{p_{1}}\left(\mathbb{R}^{n}\right) \times \cdots \times L^{p_{m}}\left(\mathbb{R}^{n}\right)$ to $L^{p}\left(\mathbb{R}^{n}\right)$ holds if $\sigma \in C^{s}\left(\mathbb{R}^{m n} \backslash\{0\}\right)$ satisfying the condition

$$
\left|\partial_{\xi}^{\alpha} \sigma(\xi)\right| \leq C_{\alpha}|\xi|^{-|\alpha|}
$$

for all multi-indice $|\alpha| \leq s$ with $s \geq 2 m n+1$ and all $1<$ $p, p_{1}, \ldots, p_{m}<\infty$ with $1 / p_{1}+\cdots+1 / p_{m}=1 / p$. Grafakos and Torres [2] improved the multiplier theorem of Coifman and Meyer to the indices $1 / m \leq p \leq 1$ by the multilinear Calderón-Zygmund operator theory in the case of $s \geq m n+1$.
An important progress in this topic was given by Tomita. Let $\Phi \in \mathcal{S}\left(\mathbb{R}^{m n}\right)$ satisfy

$$
\begin{gathered}
\operatorname{supp} \Phi \subset\left\{\left(\xi_{1}, \ldots, \xi_{m}\right): \frac{1}{2} \leq \sum_{k=1}^{m}\left|\xi_{k}\right| \leq 2\right\} ; \\
\sum_{l \in \mathbb{Z}} \Phi\left(2^{-l} \xi_{1}, \ldots, 2^{-l} \xi_{m}\right)=1,
\end{gathered}
$$

for all $\left(\xi_{1}, \ldots, \xi_{m}\right) \in \mathbb{R}^{m n} \backslash\{0\}$.

Set

$$
\begin{aligned}
\sigma_{l}\left(\xi_{1}, \ldots, \xi_{m}\right) & =\Phi\left(\xi_{1}, \ldots, \xi_{m}\right) \sigma\left(2^{l} \xi_{1}, \ldots, 2^{l} \xi_{m}\right), \\
\left\|\sigma_{l}\right\|_{W^{s}\left(\mathbb{R}^{m n}\right)}= & \left(\int_{\mathbb{R}^{m n}}\left(1+\left|\xi_{1}\right|^{2}+\cdots+\left|\xi_{m}\right|^{2}\right)^{s}\right. \\
& \left.\times\left|\widehat{\sigma}\left(\xi_{1}, \ldots, \xi_{m}\right)\right|^{2} d \vec{\xi}\right)^{1 / 2} .
\end{aligned}
$$

Tomita [3] proved that if

$$
\sup _{l \in \mathbb{Z}}\left\|\sigma_{l}\right\|_{W^{s}\left(\mathbb{R}^{m n}\right)}<\infty,
$$


for some $s \in(m n / 2, \infty)$, then $T_{\sigma}$ is bounded from $L^{p_{1}}\left(\mathbb{R}^{n}\right) \times$ $\cdots \times L^{p_{m}}\left(\mathbb{R}^{n}\right)$ to $L^{p}\left(\mathbb{R}^{n}\right)$ provided that $1<p, p_{1}, \ldots, p_{m}<$ $\infty$ and $1 / p=\sum_{k=1}^{m} 1 / p_{k}$. Grafakos and Si in [4] obtained that $T_{\sigma}$ maps from $L^{p_{1}}\left(\mathbb{R}^{n}\right) \times \cdots \times L^{p_{m}}\left(\mathbb{R}^{n}\right)$ to $L^{p}\left(\mathbb{R}^{n}\right)$, if $\sigma$ satisfies (5) and $1 / m \leq p \leq 1$. Miyachi and Tomita [5] considered the problem to find minimal smoothness condition for multilinear Fourier multiplier. Let

$$
\begin{aligned}
& \left\|\sigma_{l}\right\|_{W^{s_{1}, \ldots, s_{m}\left(\mathbb{R}^{m n}\right)}} \\
& \quad=\left(\int_{\mathbb{R}^{2 n}}\left\langle\xi_{1}\right\rangle^{2 s_{1}} \cdots\left\langle\xi_{m}\right\rangle^{2 s_{m}}\left|\widehat{\sigma}_{l}\left(\xi_{1}, \ldots, \xi_{m}\right)\right|^{2} d \vec{\xi}\right)^{1 / 2},
\end{aligned}
$$

where $\left\langle\xi_{k}\right\rangle:=\left(1+\left|\xi_{k}\right|^{2}\right)^{1 / 2}$. Miyachi and Tomita [5] proved that if

$$
\sup _{l \in \mathbb{Z}}\left\|\sigma_{l}\right\|_{W^{s_{1}, s_{2}}\left(\mathbb{R}^{2 n}\right)}<\infty
$$

for each $s_{j} \in(n / 2, n]$, then $T_{\sigma}$ is bounded from $L^{p_{1}}\left(\mathbb{R}^{n}\right) \times$ $L^{p_{2}}\left(\mathbb{R}^{n}\right)$ to $L^{p}\left(\mathbb{R}^{n}\right)$ provided that $1<p_{1}, p_{2}<\infty$, and $p>2 / 3$ with $1 / p=\sum_{k=1}^{2} 1 / p_{k}$. Moreover, they also gave minimal smoothness condition for which $T_{\sigma}$ is bounded from $H^{p_{1}}\left(\mathbb{R}^{n}\right) \times H^{p_{2}}\left(\mathbb{R}^{n}\right)$ to $L^{p}\left(\mathbb{R}^{n}\right)$.

Let $m n / 2<s \leq m n, m n / s<p_{1}, \ldots, p_{m}<\infty$, and $1 / p_{1}+$ $\cdots+1 / p_{m}=1 / p$. Fujita and Tomita [6] proved the following inequality:

$$
\left\|T_{\sigma}\left(f_{1}, \ldots, f_{m}\right)\right\|_{L^{p}\left(v_{\vec{\omega}}\right)} \leq C \prod_{k=1}^{m}\left\|f_{k}\right\|_{L^{p_{k}}\left(\omega_{k}\right)},
$$

if $\left\|\sigma_{l}\right\|_{W^{s / m, \ldots, s / m}\left(\mathbb{R}^{m n}\right)}<\infty$ and $\vec{\omega}=\left(\omega_{1}, \ldots, \omega_{m}\right) \in A_{p_{1} s /(m n)} \times$ $\cdots \times A_{p_{m} s /(m n)}$, where and in what follows $\nu_{\vec{\omega}}=\prod_{k=1}^{m} \omega_{k}^{p / p_{k}}$. $\mathrm{Li}$ et al. [7] obtained the endpoint cases. Hu and Lin [8] also obtained this result from another approach. Replacing $W^{s_{1}, \ldots, s_{m}}$ by $W^{s}$, Bui and Duong [9] and Li and Sun [10] proved that if $\vec{\omega}=\left(\omega_{1}, \ldots, \omega_{m}\right) \in A_{\left(p_{1} s /(m n), \ldots, p_{m} s /(m n)\right)}$, then (8) also holds. Jiao [11] gave a generalization of the above inequality with the class $A_{\vec{P} / \vec{Q}}$, which generalizes the class $A_{\vec{P}}$ introduced by Lerner et al. [12]. Fujita and Tomita showed a counterexample to answer the question whether the inequality (8) holds under the conditions $\vec{\omega}=\left(\omega_{1}, \ldots, \omega_{m}\right) \in$ $A_{\left(p_{1} s /(m n), \ldots, p_{m} s /(m n)\right)}$ and $\left\|\sigma_{l}\right\|_{W^{s / m, \ldots, s / m}\left(\mathbb{R}^{m n}\right)}<\infty$.

We still recall the weighted Morrey spaces which were introduced by Komori and Shirai [13]. A weight $\omega$ is a nonnegative, locally integrable function on $\mathbb{R}^{n}$. Let $1<p<$ $\infty$; a weight function $\omega$ is said to belong to the class $A_{p}$, if there is a constant $C$ such that for any cube $Q$,

$$
\left(\frac{1}{|Q|} \int_{Q} \omega(x) d x\right)\left(\frac{1}{|Q|} \int_{Q} \omega(x)^{1-p^{\prime}} d x\right)^{p-1} \leq C,
$$

and $\omega$ belongs to the class $A_{1}$, if there is a constant $C$ such that, for any cube $Q$,

$$
\frac{1}{|Q|} \int_{Q} \omega(x) d x \leq \operatorname{Cinf}_{x \in Q} \omega(x) .
$$

We denote $A_{\infty}=\cup_{p>1} A_{p}$.
Definition 1 (See [13]). Let $1 \leq p<\infty$, let $0<\kappa<1$, and let $\omega$ be a weight function on $\mathbb{R}^{n}$. The weighted Morrey space is defined by

$$
L^{p, \kappa}(\omega)=\left\{f \in L_{\mathrm{loc}}^{p}:\|f\|_{L^{p, \kappa}(\omega)}<\infty\right\},
$$

where

$$
\|f\|_{L^{p, \kappa}(\omega)}=\sup _{Q}\left(\frac{1}{\omega(Q)^{k}} \int_{Q}|f(x)|^{p} \omega(x) d x\right)^{1 / p} .
$$

Our main results can be stated as follows.

Theorem 2. Let $\sigma$ be a multiplier satisfying

$$
\left\|\sigma_{l}\right\|_{W^{s_{1}, \ldots, s_{m}\left(\mathbb{R}^{m n}\right)}}<\infty
$$

for $s_{1}, \ldots, s_{m} \in(n / 2, n]$ and let $T_{\sigma}$ be the operator defined by (1) and $0<\kappa<1$. Set $t_{j}=n / s_{j}$. If $p_{j} \in\left(t_{j}, \infty\right)$ and the weight $\omega_{j} \in A_{p_{j} / t_{j}}\left(\mathbb{R}^{n}\right)$ for $1 \leq j \leq m$ and $p \in[1, \infty)$ such that $1 / p=1 / p_{1}+\cdots+1 / p_{m}$, then

$$
\left\|T_{\sigma}\left(f_{1}, \ldots, f_{m}\right)\right\|_{L^{p, \kappa}\left(\nu_{\vec{\omega}}\right)} \leq C \prod_{j=1}^{m}\left\|f_{j}\right\|_{L^{p_{j}, \kappa}\left(\omega_{j}\right)}
$$

where $\nu_{\vec{\omega}}=\prod_{j=1}^{m} \omega_{j}^{p / p_{j}}$.

Given a multilinear Fourier multiplier operator $T_{\sigma}$ and $\vec{b}=\left(b_{1}, \ldots, b_{m}\right) \in \operatorname{BMO}\left(\mathbb{R}^{n}\right)^{m}$, we define the commutators $T_{\sigma, \Sigma \mathbf{b}}(\vec{f})(x)$ to be

$$
T_{\sigma, \Sigma \mathbf{b}}(\vec{f})(x)=\sum_{j=1}^{m}\left[b_{j}, T_{\sigma}\right]_{j}\left(f_{1}, \ldots, f_{m}\right)(x),
$$

with

$$
\begin{aligned}
{\left[b_{j}, T_{\sigma}\right]_{j}\left(f_{1}, \ldots, f_{m}\right)(x)=} & b_{j}(x) T_{\sigma}\left(f_{1}, \ldots, f_{j}, \ldots, f_{m}\right)(x) \\
& -T_{\sigma}\left(f_{1}, \ldots, b_{j} f_{j}, \ldots, f_{m}\right)(x) .
\end{aligned}
$$

Theorem 3. Let $\sigma$ be a multiplier satisfying

$$
\left\|\sigma_{l}\right\|_{W^{s_{1}, \ldots, s_{m}\left(\mathbb{R}^{m n}\right)}}<\infty
$$

for $s_{1}, \ldots, s_{m} \in(n / 2, n]$ and let $T_{\sigma}$ be the operator defined by (1) and $0<\kappa<1$. Set $t_{j}=n / s_{j}$. If $p_{j} \in\left(t_{j}, \infty\right)$ and the weight $\omega_{j} \in A_{p_{j} / t_{j}}\left(\mathbb{R}^{n}\right)$ for $1 \leq j \leq m$ and $p \in[1, \infty)$ such that $1 / p=1 / p_{1}+\cdots+1 / p_{m}$, then for any $b_{1}, \ldots, b_{m} \in B M O\left(\mathbb{R}^{n}\right)$,

$$
\begin{aligned}
& \left\|T_{\sigma, \Sigma \mathbf{b}}\left(f_{1}, \ldots, f_{m}\right)\right\|_{L^{p, \kappa}\left(\mathbb{R}^{n}, v_{\vec{\omega}}\right)} \\
& \quad \leq C\|\vec{b}\|_{B M O^{m}} \prod_{j=1}^{m}\left\|f_{j}\right\|_{L^{p_{j}, \kappa}\left(\mathbb{R}^{n}, \omega_{j}\right)},
\end{aligned}
$$

where $\|\vec{b}\|_{B M O^{m}}=\prod_{j=1}^{m}\left\|b_{j}\right\|_{B M O}$ and $\nu_{\vec{\omega}}=\prod_{j=1}^{m} \omega_{j}^{p / p_{j}}$. 
Because the regularity condition $\left\|\sigma_{l}\right\|_{W^{s}\left(\mathbb{R}^{m n}\right)}<\infty$ is stronger than that of $\left\|\sigma_{l}\right\|_{W^{s_{1}, \ldots, s_{m}}\left(\mathbb{R}^{m n}\right)}<\infty$, we have the following corollaries.

Corollary 4. Let $\sigma$ be a multiplier satisfying

$$
\left\|\sigma_{l}\right\|_{W^{s}\left(\mathbb{R}^{m n}\right)}<\infty,
$$

for $s \in(m n / 2, m n]$ and let $T_{\sigma}$ be the operator defined by (1) and $0<\kappa<1$. Set $r=\mathrm{mn} / \mathrm{s}$. If $p_{j} \in(\mathrm{mn} / \mathrm{s}, \infty)$ and the weight $\omega_{j} \in A_{p_{j} / r}\left(\mathbb{R}^{n}\right)$ for $1 \leq j \leq m$ and $p \in[1, \infty)$ such that $1 / p=1 / p_{1}+\cdots+1 / p_{m}$, then

$$
\left\|T_{\sigma}\left(f_{1}, \ldots, f_{m}\right)\right\|_{L^{p, \kappa}\left(v_{\overparen{\omega}}\right)} \leq C \prod_{j=1}^{m}\left\|f_{j}\right\|_{L^{p_{j}, \kappa}\left(\omega_{j}\right)},
$$

where $\nu_{\vec{\omega}}=\prod_{j=1}^{m} \omega_{j}^{p / p_{j}}$.

Corollary 5. Let $\sigma$ be a multiplier satisfying

$$
\left\|\sigma_{l}\right\|_{W^{s}\left(\mathbb{R}^{m n}\right)}<\infty,
$$

for $s \in(m n / 2, m n]$ and let $T_{\sigma}$ be the operator defined by (1) and $0<\kappa<1$. Set $r=\mathrm{mn} / \mathrm{s}$. If $p_{j} \in(\mathrm{mn} / \mathrm{s}, \infty)$ and the weight $\omega_{j} \in A_{p_{j} / r}\left(\mathbb{R}^{n}\right)$ for $1 \leq j \leq m$ and $p \in[1, \infty)$ such that $1 / p=1 / p_{1}+\cdots+1 / p_{m}$, then, for any $b_{1}, \ldots, b_{m} \in B M O\left(\mathbb{R}^{n}\right)$,

$$
\left\|T_{\sigma, \Sigma \mathbf{b}}\left(f_{1}, \ldots, f_{m}\right)\right\|_{L^{p, \kappa}\left(v_{\bar{\omega}}\right)} \leq C\|\vec{b}\|_{B M O^{m}} \prod_{j=1}^{m}\left\|f_{j}\right\|_{L^{p_{j}, \kappa}\left(\omega_{j}\right)},
$$

where $\|\vec{b}\|_{B M O^{m}}=\prod_{j=1}^{m}\left\|b_{j}\right\|_{B M O}$ and $v_{\vec{\omega}}=\prod_{j=1}^{m} \omega_{j}^{p / p_{j}}$.

Remark 6. For $m=1$ and $\omega \in A_{p}$, we also extend Hörmander's theorem [14] to the weighted Morrey spaces.

\section{Some Notations and Lemmas}

We begin with the definitions of Hardy-Littlewood maximal function,

$$
M f(x)=\sup _{\mathrm{Q} \ni x} \frac{1}{|Q|} \int_{Q}|f(y)| d y
$$

and of the sharp maximal function,

$$
M^{\sharp}(f)(x)=\sup _{\mathrm{Q} \ni x} \inf _{c \in \mathbb{R}} \frac{1}{|Q|} \int_{\mathrm{Q}}|f(y)-c| d y .
$$

For $\delta>0$, we also define the following maximal functions $M_{\delta}(f)=M\left(|f|^{\delta}\right)^{1 / \delta}$ and $M_{\delta}^{\sharp}(f)=M^{\sharp}\left(|f|^{\delta}\right)^{1 / \delta}$. The following classical result belongs to Fefferman and Stein [15].

Lemma 7. Let $0<p, \delta<\infty$, and $\omega \in A_{\infty}$. Then there exists some constant $C_{n, p, \delta, \omega}$ such that

$$
\left\|M_{\delta}(f)\right\|_{L^{p}(\omega)} \leq C_{n, p, \delta, \omega}\left\|M_{\delta}^{\sharp}(f)\right\|_{L^{p}(\omega)} .
$$

Similarly, we have the responding lemma on weighted Morrey spaces as a consequent result.

Lemma 8. Let $0<\kappa<1,0<p, \delta<\infty$, and $\omega \in A_{\infty}$. Then there exists some constant $C_{n, p, \delta, \omega}$ such that

$$
\left\|M_{\delta}(f)\right\|_{L^{p, \kappa}(\omega)} \leq C_{n, p, \delta, \omega}\left\|M_{\delta}^{\sharp}(f)\right\|_{L^{p, \kappa}(\omega)} .
$$

For $\vec{f}=\left(f_{1}, \ldots, f_{m}\right), r_{i}>0, i=1, \ldots, m$, and set $\vec{r}=$ $\left(r_{1}, \ldots, r_{m}\right)$, we define

$$
\mathscr{M}_{\vec{r}}(\vec{f})(x)=\sup _{\mathrm{Q} \ni x} \prod_{j=1}^{m}\left(\frac{1}{|Q|} \int_{Q}\left|f_{l}\left(y_{j}\right)\right|^{r_{j}} d y_{j}\right)^{1 / r_{j}} .
$$

This maximal function is the generalization of $\mathscr{M}$ which is introduced by Lerner et al. [12], we refer to [11] for some properties of it. The following lemma is the special example of [11, Theorem 2.1].

Lemma 9. Let $p_{1}, \ldots, p_{m}, p \in(0, \infty), r_{j} \in\left(0, p_{j}\right)$, and $\omega_{j} \in$ $A_{p_{j} / r_{j}}$ for $1 \leq j \leq m$ and $1 / p_{1}+\cdots+1 / p_{m}=1 / p$. Then we have

$$
\left\|\mathscr{M}_{\vec{r}}(\vec{f})\right\|_{L^{p}\left(\nu_{\vec{\omega}}\right)} \leq C \prod_{j=1}^{m}\left\|f_{j}\right\|_{L^{p_{j}}\left(\omega_{j}\right)}
$$

and if at least one $r_{l}=p_{l}$, then

$$
\left\|\mathscr{M}_{\vec{r}}(\vec{f})\right\|_{L^{p, \infty}\left(v_{\vec{\omega}}\right)} \leq C \prod_{j=1}^{m}\left\|f_{j}\right\|_{L^{p_{j}}\left(\omega_{j}\right)}
$$

where $v_{\vec{\omega}}=\prod_{l=1}^{m} \omega_{l}^{p / p_{l}}$.

Lemma 10. Let $\kappa \in(0,1), p_{1}, \ldots, p_{m}, p \in(0, \infty), r_{j} \in\left(0, p_{l}\right)$, and $\omega_{j} \in A_{p_{j} / r_{j}}$ for $1 \leq j \leq m$ and $1 / p_{1}+\cdots+1 / p_{m}=1 / p$. Then we have

$$
\left\|M_{\vec{r}}(\vec{f})\right\|_{L^{p, \kappa}\left(v_{\vec{\omega}}\right)} \leq C \prod_{j=1}^{m}\left\|f_{j}\right\|_{L^{p j, \kappa}\left(\omega_{j}\right)}
$$

Proof. From [11], there exists some $q \in(0,1)$ such that

$$
\mathscr{M}_{\vec{r}}(\vec{f})(x) \leq C \prod_{j=1}^{m}\left\{M_{\nu_{\vec{\omega}}}^{c}\left(\left(\frac{\left|f_{j}\right|^{p_{j}} \omega_{j}}{v_{\vec{\omega}}}\right)^{q}\right)(x)\right\}^{1 /\left(q p_{j}\right)},
$$


where $M_{\nu_{\vec{\omega}}}^{c}$ is the weighted centered maximal operator. Then by the Hölder inequality and [13, Theorem 3.1$]$, we get

$$
\begin{aligned}
& \left\|M_{\vec{r}}(\vec{f})(x)\right\|_{L^{p, k}\left(v_{\vec{\omega}}\right)} \\
& \leq C\left\|\prod_{j=1}^{m}\left\{M_{v_{\vec{\omega}}}^{c}\left(\left[\frac{\left|f_{j}\right|^{p_{j}} \omega_{j}}{v_{\vec{\omega}}}\right]^{q}\right)\right\}^{1 /\left(q p_{j}\right)}\right\|_{L^{p, k}\left(v_{\vec{\omega}}\right)} \\
& \leq C \prod_{j=1}^{m}\left\|\left\{M_{v_{\vec{\omega}}}^{c}\left(\left[\frac{\left|f_{j}\right|^{p_{j}} \omega_{j}}{v_{\vec{\omega}}}\right]^{q}\right)\right\}^{1 /\left(q p_{j}\right)}\right\| \\
& \leq C \prod_{j=1}^{m}\left\|M_{v_{\vec{\omega}}^{c}}^{c}\left(\left[\frac{\left|f_{j}\right|^{p_{j}} \omega_{j}}{v_{\vec{\omega}}}\right]^{q}\right)\right\|_{L^{p_{j}, k}\left(v_{\vec{\omega}}\right)}^{1 /\left(q p_{j}\right)} \\
& \leq C \prod_{j=1}^{m}\left\|\left(\frac{\left|f_{j}\right|^{p_{j}, k} \omega_{j}}{v_{\vec{\omega}}}\right)^{q}\right\|_{\left.\nu_{\vec{\omega}}\right)}^{1 /\left(q p_{j}\right)} \\
& \leq C \prod_{j=1}^{m}\left\|f_{j}\right\|_{L^{p_{j}, k, k}\left(\omega_{j}\right)}
\end{aligned}
$$

Lemma 11 (See [6]). Let $1<p_{1}, \ldots, p_{m}<\infty$ and $1 / p=$ $1 / p_{1}+\cdots+1 / p_{m}$. Suppose that $\sigma \in L^{\infty}\left(\mathbb{R}^{m n}\right)$ satisfies

$$
\left\|\sigma_{l}\right\|_{W^{s_{1}, \ldots, s_{m}\left(\mathbb{R}^{m n}\right)}}<\infty .
$$

Then $T_{\sigma}$ is bounded from $L^{p_{1}}\left(\mathbb{R}^{n}\right) \times \cdots \times L^{p_{m}}\left(\mathbb{R}^{n}\right)$ to $L^{p}\left(\mathbb{R}^{n}\right)$.

For $q_{1}, \ldots, q_{m} \in(0, \infty)$ and $s_{1}, \ldots, s_{m} \in \mathbb{R}$, the weighted Lebesgue space of mixed type $L^{\left(q_{1}, \ldots, q_{m}\right)}\left(\omega_{\left(s_{1}, \ldots, s_{m}\right)}\right)$ is defined by the norm

$$
\begin{aligned}
& \|F\|_{L^{\left(q_{1}, \ldots, q_{m}\right)}\left(\omega_{\left(s_{1}, \ldots, s_{m}\right)}\right)} \\
& =\left[\int _ { \mathbb { R } ^ { n } } \ldots \left\{\int_{\mathbb{R}^{n}}\left(\int_{\mathbb{R}^{n}}|F(x)|^{q_{1}}\left\langle x_{1}\right\rangle^{s_{1}} d x_{1}\right)^{q_{2} / q_{1}}\right.\right. \\
& \left.\left.\quad \times\left\langle x_{2}\right\rangle^{s_{2}} d x_{2}\right\}^{q_{3} / q_{2}} \cdots\left\langle x_{m}\right\rangle^{s_{m}} d x_{m}\right]^{1 / q_{m}} .
\end{aligned}
$$

Lemma 12 (See [6]). Let $r>0,2 \leq q_{j}<\infty$, and $s_{j} \geq 0$ for $1 \leq j \leq m$. Then there exists a constant $C>0$ such that

$$
\|\widehat{F}\|_{L^{\left(q_{1}, \ldots, q_{m}\right)}\left(\omega_{\left(s_{1}, \ldots, s_{m}\right)}\right)} \leq C\|F\|_{W^{s_{1} / q_{1}, \ldots, s_{m} / q_{m}}}
$$

for all $F \in W^{s_{1} / q_{1}, \ldots, s_{m} / q_{m}}\left(\mathbb{R}^{m n}\right)$ with $\operatorname{supp} F \subset\left\{\left|x_{1}\right|^{2} \cdots+\right.$ $\left.\left|x_{m}\right|^{2} \leq r\right\}$.

By the reverse Hölder inequality, we have the following lemma.
Lemma 13. Assume that $\vec{\omega} \in \prod_{j=1}^{m} A_{p_{j}}$, with $1<p_{1}, \ldots, p_{m}<$ $\infty$. Let $n / 2<s_{j} \leq n$; then there exist constants $1<\epsilon_{j}<$ $\min \left\{p_{j}, s_{j} /\left(s_{j}-1\right), 2 s_{j} / n\right\}$ such that $\omega_{j} \in A_{p_{j} / \epsilon_{j}}$.

The following lemma is the key to our main results.

Lemma 14. Let " $\sigma$ " be a multplier satisfying

$$
\left\|\sigma_{l}\right\|_{W^{\left(s_{1}, \ldots, s_{m}\right)}\left(\mathbb{R}^{n}\right)}<\infty
$$

for $s_{1}, \ldots, s_{m} \in(n / 2, n]$ and let $T_{\sigma}$ be the operator defined by (1). If $1<p_{j}<\infty, t_{j}=n / s_{j}$ and $0<\delta<r / m$, where $1 / r=1 / r_{1}+\cdots+1 / r_{m}, r_{j}=\epsilon_{j} t_{j}$ and $1<\epsilon_{j}<\min \left\{p_{j}, s_{j} /\left(s_{j}-\right.\right.$ 1), $\left.2 s_{j} / n\right\}$. Then for all $\vec{f} \in L^{p_{1}}\left(\mathbb{R}^{n}\right) \times \cdots \times L^{p_{m}}\left(\mathbb{R}^{n}\right)$ with $r_{j} \leq$ $p_{j}<\infty$ for $1 \leq j \leq m$,

$$
M_{\delta}^{\sharp}\left(T_{\sigma}(\vec{f})\right)(x) \leq C \mathscr{M}_{\vec{r}}(\vec{f})(x),
$$

where $\vec{r}=\left(r_{1}, \ldots, r_{m}\right)$.

Proof. By Lemma $13,1<t_{j} \epsilon_{j} \leq 2$; then $r_{j} / m \leq 1$. Fix a point $x$ and a cube $Q$ such that $x \in Q$. It suffices to prove that

$$
\left(\frac{1}{|Q|} \int_{Q}\left|T_{\sigma}(\vec{f})(z)-c_{Q}\right|^{\delta} d z\right)^{1 / \delta} \leq C \mathscr{M}_{\vec{r}}(\vec{f})(x),
$$

for some constant $c_{Q}$. We decompose $f_{j}=f_{j}^{0}+f_{j}^{\infty}$ with $f_{j}^{0}=$ $f_{j} \chi_{Q^{\star}}$ for all $j=1, \ldots, m$ and $Q^{\star}=4 \sqrt{n} Q$. Then

$$
\begin{aligned}
\prod_{j=1}^{m} f_{j}\left(y_{j}\right) & =\prod_{j=1}^{m}\left(f_{j}^{0}\left(y_{j}\right)+f_{j}^{\infty}\left(y_{j}\right)\right) \\
& =\sum_{\alpha_{1}, \ldots, \alpha_{m} \in\{0, \infty\}} f_{1}^{\alpha_{1}}\left(y_{1}\right) \cdots f_{m}^{\alpha_{m}}\left(y_{m}\right) \\
& =\prod_{j=1}^{m} f_{j}^{0}\left(y_{j}\right)+\sum_{\alpha_{1}, \ldots, \alpha_{m} \in \mathcal{I}} f_{1}^{\alpha_{1}}\left(y_{1}\right) \cdots f_{m}^{\alpha_{m}}\left(y_{m}\right),
\end{aligned}
$$

where $\mathscr{I}=\left\{\alpha_{1}, \ldots, \alpha_{m}\right.$ : there is at least one $\left.\alpha_{j} \neq 0\right\}$. Then we can write

$$
\begin{aligned}
T_{\sigma}(\vec{f})(z) & =T_{\sigma}\left(\vec{f}^{0}\right)(z)+\sum_{\alpha_{1}, \ldots, \alpha_{m} \in \mathscr{I}} T_{\sigma}\left(f_{1}^{\alpha_{1}} \cdots f_{m}^{\alpha_{m}}\right)(z) \\
& :=I+I I .
\end{aligned}
$$

Applying Kolmogorov's inequality to $I$, we have

$$
\begin{aligned}
& \left(\frac{1}{|Q|} \int_{Q}\left|T_{\sigma}\left(\vec{f}^{0}\right)(z)\right|^{\delta} d z\right)^{1 / \delta} \\
& \quad \leq C\left\|T_{\sigma}\left(\vec{f}^{0}\right)\right\|_{L^{r, \infty}(\mathrm{Q}, d x /|\mathrm{Q}|)} \\
& \quad \leq C \prod_{j=1}^{m}\left(\frac{1}{\left|Q^{\star}\right|} \int_{\mathrm{Q}^{\star}}\left|f_{j}\left(y_{j}\right)\right|^{r_{l}} d y_{j}\right)^{r_{j}} \\
& \quad \leq C \mathscr{M}_{\vec{r}}(\vec{f})(x),
\end{aligned}
$$

since $T_{\sigma}$ is bounded from $L^{r_{1}} \times \cdots \times L^{r_{m}}$ to $L^{r}$ by Lemma 11 . 
Taking

$$
c_{Q}=\sum_{\alpha_{1}, \ldots, \alpha_{m} \in \mathscr{F}} T_{\sigma}\left(f_{1}^{\alpha_{1}} \cdots f_{m}^{\alpha_{m}}\right)(x)
$$

we claim that, for any $z \in Q$,

$$
\begin{aligned}
& \sum_{\alpha_{1}, \ldots, \alpha_{m} \in \mathscr{I}}\left|T_{\sigma}\left(f_{1}^{\alpha_{1}} \cdots f_{m}^{\alpha_{m}}\right)(z)-T_{\sigma}\left(f_{1}^{\alpha_{1}} \cdots f_{m}^{\alpha_{m}}\right)(x)\right| \\
& \leq C \mathscr{M}_{\vec{r}}(\vec{f})(x) .
\end{aligned}
$$

$$
\begin{aligned}
& \left|T_{\sigma}\left(f_{1}^{\infty} \cdots f_{m}^{\infty}\right)(z)-T_{\sigma}\left(f_{1}^{\infty} \cdots f_{m}^{\infty}\right)(x)\right| \\
& \leq \sum_{l \in \mathbb{Z}}\left|T_{\sigma_{l}}\left(f_{1}^{\infty} \cdots f_{m}^{\infty}\right)(z)-T_{\sigma_{l}}\left(f_{1}^{\infty} \cdots f_{m}^{\infty}\right)(x)\right| \leq \sum_{l \in \mathbb{Z}} \int_{\mathbb{R}^{m n} \backslash\left(Q^{\star}\right)^{m}}\left|W_{l}\left(x, z ; y_{1}, \ldots, y_{m}\right)\right| \prod_{j=1}^{m} f_{j}\left(y_{j}\right) d \vec{y} \\
& \leq \sum_{l \in \mathbb{Z}} \sum_{k=0}^{\infty} \int_{\left(2^{k+1} Q^{\star} \backslash 2^{k} Q^{\star}\right)^{m}}\left|W_{l}\left(x, z ; y_{1}, \ldots, y_{m}\right)\right| \prod_{j=1}^{m} f_{j}\left(y_{j}\right) d \vec{y} \\
& \leq \sum_{k=0}^{\infty} \sum_{l \in \mathbb{Z}} \prod_{j=1}^{m}\left(\int_{2^{k+1} Q^{\star}}\left|f_{j}\left(y_{j}\right)\right|^{r_{j}} d y_{j}\right)^{1 / r_{j}} \\
& \times\left(\int_{\left(2^{k+1} Q^{\star}\right) \backslash\left(2^{k} Q^{\star}\right)}\left(\int_{\left(2^{k+1} Q^{\star}\right) \backslash\left(2^{k} Q^{\star}\right)} \cdots\left(\int_{\left(2^{k+1} Q^{\star}\right) \backslash\left(2^{k} Q^{\star}\right)}\left|W_{l}\left(x, z ; y_{1}, \ldots, y_{m}\right)\right|^{r_{1}^{\prime}} d y_{1}\right)^{r_{2}^{\prime} / r_{1}^{\prime}} \ldots\right)^{r_{m}^{\prime} / r_{m-1}^{\prime}} d y_{m}\right)^{1 / r_{m}^{\prime}} \\
& :=\sum_{k=0}^{\infty} \sum_{l \in \mathbb{Z}} \prod_{j=1}^{m}\left(\int_{2^{k+1} Q^{\star}}\left|f_{j}\left(y_{j}\right)\right|^{r_{j}} d y_{j}\right)^{1 / r_{j}} I I_{k, l}^{\infty, \ldots, \infty} .
\end{aligned}
$$

Denote $h=z-x$ and $\widetilde{Q}=x-Q^{\star}$; it follows from Lemma 12

that

$I I_{k, l}^{\infty, \ldots, \infty}$

$=\left(\int_{\left(2^{k+1} \widetilde{\mathrm{Q}}\right) \backslash\left(2^{k} \widetilde{\mathrm{Q}}\right)}\left(\int_{\left(2^{k+1} \widetilde{\mathrm{Q}}\right) \backslash\left(2^{k} \widetilde{\mathrm{Q}}\right)} \cdots\left(\int_{\left(2^{k+1} \widetilde{\mathrm{Q}}\right) \backslash\left(2^{k} \widetilde{\mathrm{Q}}\right)}\left|\check{\sigma}_{l}\left(h+y_{1}, \ldots, h+y_{m}\right)-\check{\sigma}_{l}\left(y_{1}, \ldots, y_{m}\right)\right|^{r_{1}^{\prime}} d y_{1}\right)^{r_{2}^{\prime} / r_{1}^{\prime}} \ldots\right)^{r_{m}^{\prime} / r_{m-1}^{\prime}} d y_{m}\right)^{1 / r_{m}^{\prime}}$

$\leq 2\left(\int_{c_{1} 2^{k} \ell(Q) \leq\left|y_{m}\right|<c_{2} 2^{k+1} \ell(Q)}\left(\int_{c_{1} 2^{k} \ell(Q) \leq\left|y_{m-1}\right|<c_{2} 2^{k+1} \ell(Q)} \cdots\left(\int_{c_{1} 2^{k} \ell(Q) \leq\left|y_{1}\right|<c_{2} 2^{k+1} \ell(Q)}\left|\check{\sigma}_{l}\left(y_{1}, \ldots, y_{m}\right)\right|^{r_{1}^{\prime}} d y_{1}\right)^{r_{2}^{\prime} / r_{1}^{\prime}} \ldots\right)^{r_{m}^{\prime} / r_{m-1}^{\prime}} d y_{m}\right)^{1 / r_{m}^{\prime}}$ $\leq C\left(2^{k} l(Q)\right)^{-\left(s_{1}+\cdots+s_{m}\right)}$

$\times\left(\int_{c_{1} 2^{k} \ell(\mathrm{Q}) \leq\left|y_{m}\right|<c_{2} 2^{k+1} \ell(\mathrm{Q})}\left(\int_{\mathcal{c}_{1} 2^{k} \ell(\mathrm{Q}) \leq\left|y_{m-1}\right|<c_{2} 2^{k+1} \ell(\mathrm{Q})} \cdots\left(\int_{c_{1} 2^{k} \ell(\mathrm{Q}) \leq\left|y_{1}\right|<c_{2} 2^{k+1} \ell(\mathrm{Q})}\left|\check{\sigma}_{l}\left(y_{1}, \ldots, y_{m}\right)\right|^{r_{1}^{\prime}}\left\langle y_{1}\right\rangle^{s_{1} r_{1}^{\prime}} d y_{1}\right)^{r_{2}^{\prime} / r_{1}^{\prime}} \ldots\right)^{r_{m}^{\prime} / r_{m-1}^{\prime}}\right.$ $\left.\times\left\langle y_{m}\right\rangle^{s_{m} r_{m}^{\prime}} d y_{m}\right)^{1 / r_{m}^{\prime}}$ 


$$
\begin{aligned}
& \leq C\left(2^{k} l(Q)\right)^{-\left(s_{1}+\cdots+s_{m}\right)} 2^{l\left(s_{1}+\cdots+s_{m}\right)} \\
& \times\left(\int_{c_{1} 2^{k} \ell(Q) \leq\left|y_{m}\right|<c_{2} 2^{k+1} \ell(Q)}\right. \\
& \left(\int_{c_{1} 2^{k} \ell(\mathrm{Q}) \leq\left|y_{m-1}\right|<c_{2} 2^{k+1} \ell(\mathrm{Q})} \cdots\left(\int_{c_{1} 2^{k} \ell(\mathrm{Q}) \leq\left|y_{1}\right|<c_{2} 2^{k+1} \ell(\mathrm{Q})}\left|2^{-l m n} \check{\sigma}_{l}\left(2^{-l} y_{1}, \ldots, 2^{-l} y_{m}\right)\right|^{r_{1}^{\prime}}\left\langle 2^{-l} y_{1}\right\rangle^{s_{1} r_{1}^{\prime}} d y_{1}\right)^{r_{2}^{\prime} / r_{1}^{\prime}} \cdots\right)^{r_{m}^{\prime} / r_{m-1}^{\prime}} \\
& \left.\times\left\langle 2^{-l} y_{m}\right\rangle^{s_{m} r_{m}^{\prime}} d y_{m}\right)^{1 / r_{m}^{\prime}} \\
& \leq C\left(2^{k} l(Q)\right)^{-\left(s_{1}+\cdots+s_{m}\right)} 2^{l\left(s_{1}+\cdots+s_{m}\right)} 2^{-l m n} 2^{-l\left(n / r_{1}^{\prime}+\cdots+n / r_{m}^{\prime}\right)} \\
& \times\left(\int_{c_{1} 2^{k} \ell(Q) \leq\left|y_{m}\right|<c_{2} 2^{k+1} \ell(Q)}\left(\int_{c_{1} 2^{k} \ell(Q) \leq\left|y_{m-1}\right|<c_{2} 2^{k+1} \ell(Q)} \cdots\left(\int_{c_{1} 2^{k} \ell(Q) \leq\left|y_{1}\right|<c_{2} 2^{k+1} \ell(Q)}\left|\check{\sigma}_{l}\left(z_{1}, \ldots, z_{m}\right)\right|^{r_{1}^{\prime}}\left\langle z_{1}\right\rangle^{s_{1} r_{1}^{\prime}} d z_{1}\right)^{r_{2}^{\prime} / r_{1}^{\prime}} \ldots\right)^{r_{m}^{\prime} / r_{m-1}^{\prime}}\right. \\
& \left.\times\left\langle z_{m}\right\rangle^{s_{m} r_{m}^{\prime}} d z_{m}\right)^{1 / r_{m}^{\prime}} \\
& \leq C\left(2^{k} l(Q)\right)^{-\left(s_{1}+\cdots+s_{m}\right)} 2^{-l\left(n / r_{1}+\cdots+n / r_{m}-s_{1}-\cdots-s_{m}\right)}\left\|\sigma_{l}\right\|_{W^{s_{1}, \ldots, s_{m}}} .
\end{aligned}
$$

Given that $2^{l_{0}} \leq l(Q) \leq 2^{l_{0}+1}$, then we have that

$$
\begin{aligned}
& \sum_{l<l_{0}} I I_{k, l}^{\infty, \ldots, \infty} \\
& \quad \leq \operatorname{csup}_{l}\left\|\sigma_{l}\right\|_{W^{s_{1}, \ldots, s_{m}}} \sum_{l<l_{0}}\left(2^{k} l(Q)\right)^{-\left(s_{1}+\cdots+s_{m}\right)}
\end{aligned}
$$

$$
\begin{aligned}
& \times 2^{-l\left(n / r_{1}+\cdots+n / r_{m}-s_{1}-\cdots-s_{m}\right)} \\
\leq & \operatorname{Csup}\left\|\sigma_{l}\right\|_{W^{s_{1}, \cdots, s_{m}}} 2^{-k\left(s_{1}+\cdots+s_{m}\right)} l(Q)^{-\left(n / r_{1}+\cdots+n / r_{m}\right)} .
\end{aligned}
$$

On the other hand, a similar process follows in [10]; we get that

$$
\begin{aligned}
& I I_{k, l}^{\infty, \ldots, \infty}=\left(\int_{\left(2^{k+1} \widetilde{\mathrm{Q}}\right) \backslash\left(2^{k} \widetilde{\mathrm{Q}}\right)}\left(\int_{\left(2^{k+1} \widetilde{\mathrm{Q}}\right) \backslash\left(2^{k} \widetilde{\mathrm{Q}}\right)} \cdots\left(\int_{\left(2^{k+1} \widetilde{\mathrm{Q}}\right) \backslash\left(2^{k} \widetilde{\mathrm{Q}}\right)}\left|\check{\sigma}_{l}\left(h+y_{1}, \ldots, h+y_{m}\right)-\check{\sigma}_{l}\left(y_{1}, \ldots, y_{m}\right)\right|^{r_{1}^{\prime}} d y_{1}\right)^{r_{2}^{\prime} / r_{1}^{\prime}} \ldots\right)^{r_{m}^{\prime} / r_{m-1}^{\prime}} d y_{m}\right)^{1 / r_{m}^{\prime}} \\
& \leq\left(\int _ { c _ { 1 } 2 ^ { k } \ell ( Q ) \leq | y _ { m } | < c _ { 2 } 2 ^ { k + 1 } \ell ( Q ) } \left(\int_{c_{1} 2^{k} \ell(Q) \leq\left|y_{m-1}\right|<c_{2} 2^{k+1} \ell(Q)} \cdots\right.\right. \\
& \left.\left.\left(\int_{c_{1} 2^{k} \ell(Q) \leq\left|y_{1}\right|<c_{2} 2^{k+1} \ell(\mathrm{Q})}\left(\int_{0}^{1}\left|\vec{h} \cdot \nabla \check{\sigma}_{l}\left(y_{1}+\theta h, \ldots, y_{m}+\theta h\right)\right| d \theta\right)^{r_{1}^{\prime}} d y_{1}\right)^{r_{2}^{\prime} / r_{1}^{\prime}} \cdots\right)^{r_{m}^{\prime} / r_{m-1}^{\prime}} d y_{m}\right)^{1 / r_{m}^{\prime}}
\end{aligned}
$$




$$
\begin{aligned}
& \leq \int_{0}^{1}\left(\int_{c_{1} 2^{k} \ell(Q) \leq\left|y_{m}\right|<c_{2}{ }^{k+1} \ell(Q)}\right. \\
& \left.\quad\left(\int_{c_{1} 2^{k} \ell(Q) \leq\left|y_{m-1}\right|<c_{2} 2^{k+1} \ell(Q)} \ldots\left(\int_{c_{1} 2^{k} \ell(Q) \leq\left|y_{1}\right|<c_{2} 2^{k+1} \ell(Q)}\left|\vec{h} \cdot \nabla \check{\sigma}_{l}\left(y_{1}+\theta h, \ldots, y_{m}+\theta h\right)\right|^{r_{1}^{\prime}} d y_{1}\right)^{r_{2}^{\prime} / r_{1}^{\prime}} \ldots\right)^{r_{m}^{\prime} / r_{m-1}^{\prime}} d y_{m}\right)^{1 / r_{m}^{\prime}} d \theta \\
& \leq\left(\int_{c_{1} 2^{k} \ell(Q) \leq\left|y_{m}\right|<c_{2} 2^{k+1} \ell(Q)}\left(\int_{c_{1} 2^{k} \ell(Q) \leq\left|y_{m-1}\right|<c_{2} 2^{k+1} \ell(Q)} \ldots\left(\int_{c_{1} 2^{k} \ell(Q) \leq\left|y_{1}\right|<c_{2} 2^{k+1} \ell(Q)}\left|\vec{h} \cdot \nabla \check{\sigma}_{l}\left(y_{1}, \ldots, y_{m}\right)\right|^{r_{1}^{\prime}} d y_{1}\right)^{r_{2}^{\prime} / r_{1}^{\prime}} \ldots\right)^{r_{m}^{\prime} / r_{m-1}^{\prime}} d y_{m}\right)^{1 / r_{m}^{\prime}},
\end{aligned}
$$

where $\vec{h}=(h, \ldots, h) \in \mathbb{R}^{m n}$. Since

we have

$\vec{h} \cdot \nabla \check{\sigma}_{l}\left(y_{1}, \ldots, y_{m}\right)=\sum_{j=1}^{m} h_{j} \partial_{j} \nabla \check{\sigma}_{l}\left(y_{1}, \ldots, y_{m}\right)$,

$$
\begin{aligned}
& I I_{k, l}^{\infty, \ldots, \infty} \\
& \leq \sum_{j=1}^{m} \ell(Q)\left(\int_{c_{1} 2^{k} \ell(Q) \leq\left|y_{m}\right| c_{2} 2^{k+1} \ell(Q)}\left(\int_{c_{1} 2^{k} \ell(Q) \leq 1 y_{m-1} \mid<c_{2} 2^{k+1} \ell(Q)} \ldots\left(\int_{c_{1} 2^{k} \ell(Q) \leq\left|y_{1}\right|<c_{2} 2^{k+1} \ell(Q)}\left|\partial_{j} \cdot \check{\sigma}_{l}\left(y_{1}, \ldots, y_{m}\right)\right|^{r_{1}^{\prime}} d y_{1}\right)^{r_{2}^{\prime} / r_{1}^{\prime}} \ldots\right)^{r_{m}^{\prime} / r_{m-1}^{\prime}} d y_{m}\right)^{1 / r_{m}^{\prime}} \\
& \leq \sum_{j=1}^{m} \ell(Q)\left(2^{k} l(Q)\right)^{-\left(s_{1}+\cdots+s_{m}\right)} \\
& \times\left(\int_{c_{1} 2^{k} \ell(Q) \leq 1 y_{m} \mid<c_{2} 2^{k+1} \ell(Q)}\left(\int_{c_{1} 2^{k} \ell(Q) \leq\left|y_{m-1}\right|<c_{2} 2^{k+1} \ell(Q)} \ldots\left(\int_{c_{1} 2^{k} \ell(Q) \leq\left|y_{1}\right|<c_{2} 2^{k+1} \ell(Q)}\left|\partial_{j} \check{\sigma}_{l}\left(y_{1}, \ldots, y_{m}\right)\right|^{r_{1}^{\prime}}\left\langle y_{1}\right\rangle^{s_{1} r_{1}^{\prime}} d y_{1}\right)^{r_{2}^{\prime} / r_{1}^{\prime}} \ldots\right)^{r_{m}^{\prime} / r_{m-1}^{\prime}}\right. \\
& \left.\times\left\langle y_{m}\right\rangle^{s_{m} r_{m}^{\prime}} d y_{m}\right)^{1 / r_{m}^{\prime}} \\
& \leq C\left(2^{k} l(Q)\right)^{-\left(s_{1}+\cdots+s_{m}\right)} 2^{l\left(s_{1}+\cdots+s_{m}\right)} \\
& \times\left(\int_{\mathcal{C}_{1} 2^{k} \ell(Q) \leq\left|y_{m}\right| c_{2} 2^{k+1} \ell(Q)}\right. \\
& \left(\int_{c_{1} 2^{k} \ell(Q) \leq\left|y_{m-1}\right|<c_{2} 2^{k+1} \ell(Q)} \cdots\left(\int_{c_{1} 2^{2} \ell(Q) \leq\left|y_{1}\right|<c_{2} 2^{k+1} \ell(Q)}\left|2^{-l m n} \cdot \partial_{j} \check{\sigma}_{l}\left(2^{-l} y_{1}, \ldots, 2^{-l} y_{m}\right)\right|^{r_{1}^{\prime}}\left\langle 2^{-l} y_{1}\right\rangle^{s_{1} r_{1}^{\prime}} d y_{1}\right)^{r_{2}^{\prime} / r_{1}^{\prime}} \cdots\right)^{r_{m}^{\prime} / r_{m-1}^{\prime}} \\
& \left.\times\left\langle 2^{-l} y_{m}\right\rangle^{s_{m} r_{m}^{\prime}} d y_{m}\right)^{1 / r_{m}^{\prime}}
\end{aligned}
$$




$$
\begin{aligned}
& \leq C\left(2^{k} l(Q)\right)^{-\left(s_{1}+\cdots+s_{m}\right)} 2^{l\left(s_{1}+\cdots+s_{m}\right)} 2^{-l m n} 2^{-l\left(n / r_{1}^{\prime}+\cdots+n / r_{m}^{\prime}\right)} \\
& \quad \times\left(\int_{c_{1} 2^{k} \ell(Q) \leq\left|y_{m}\right|<c_{2} 2^{k+1} \ell(Q)}\left(\int_{c_{1} 2^{k} \ell(Q) \leq\left|y_{m-1}\right|<c_{2} 2^{k+1} \ell(Q)} \cdots\left(\int_{c_{1} 2^{k} \ell(Q) \leq\left|y_{1}\right|<c_{2} 2^{k+1} \ell(Q)}\left|\partial_{j} \check{\sigma}_{l}\left(z_{1}, \ldots, z_{m}\right)\right|^{r_{1}^{\prime}}\left\langle z_{1}\right\rangle^{s_{1} r_{1}^{\prime}} d z_{1}\right)^{r_{2}^{\prime} / r_{1}^{\prime}} \cdots\right)^{r_{m}^{\prime} / r_{m-1}^{\prime}}\right. \\
& \left.\quad \times\left\langle z_{m}\right\rangle^{s_{m} r_{m}^{\prime}} d z_{m}\right)^{1 / r_{m}^{\prime}} \\
& \leq C\left(2^{k} l(Q)\right)^{-\left(s_{1}+\cdots+s_{m}\right)} 2^{-l\left(n / r_{1}+\cdots+n / r_{m}+1-s_{1}-\cdots-s_{m}\right)}\left\|\sigma_{l}\right\|_{W^{s_{1} \ldots \ldots, s_{m}} .} .
\end{aligned}
$$

From Lemma $13, n / r_{1}+\cdots+n / r_{m}>s_{1}+\cdots+s_{m}-1$, it deduces that

$$
\sum_{l \geq l_{0}} I I_{k, l}^{\infty, \ldots, \infty} \leq \sup _{l}\left\|\sigma_{l}\right\|_{W^{s_{1}, \ldots, s_{m}}} 2^{-k\left(s_{1}+\cdots+s_{m}\right)} l(Q)^{-\left(n / r_{1}+\cdots+n / r_{m}\right)} .
$$

So

$$
\left|T_{\sigma}\left(f_{1}^{\infty} \cdots f_{m}^{\infty}\right)(z)-T_{\sigma}\left(f_{1}^{\infty} \cdots f_{m}^{\infty}\right)(x)\right|
$$$$
\left|T_{\sigma}\left(f_{1}^{\alpha_{1}}, \ldots, f_{m}^{\alpha_{m}}\right)(z)-T_{\sigma}\left(f_{1}^{\alpha_{1}}, \ldots, f_{m}^{\alpha_{m}}\right)(x)\right|
$$$$
\leq \sum_{k=0}^{\infty} \sum_{l \in \mathbb{Z}} \prod_{j=1}^{m}\left(\int_{2^{k+1} Q^{\star}}\left|f_{j}\left(y_{j}\right)\right|^{r_{j}} d \vec{y}\right)^{1 / r_{j}}
$$$$
\times\left(\int_{2^{k+1} Q^{\star} \backslash 2^{k} Q^{\star}} \cdots\left(\int_{2^{k+1} Q^{\star} \backslash 2^{k} Q^{\star}}\left(\int_{Q^{\star}} \cdots\left(\int_{Q^{\star}}\left|W_{l}\left(x, z ; y_{1}, \ldots, y_{m}\right)\right|^{r_{1}^{\prime}} d y_{1}\right)^{r_{2}^{\prime} / r_{1}^{\prime}} \cdots d y_{\gamma}\right)^{r_{\gamma+1}^{\prime} / r_{\gamma}^{\prime}} d y_{\gamma+1}\right)^{r_{\gamma+2}^{\prime} / r_{\gamma+1}^{\prime}} \cdots d y_{m}\right)^{1 / r_{m}^{\prime}}
$$

The same argument as the case $\alpha_{1}=\cdots=\alpha_{m}=\infty$ computes that

This completes the proof.

Lemma 15. "Let $\sigma$ ” be a multplier satisfying

$$
\left\|\sigma_{l}\right\|_{W^{\left(s_{1}, \cdots, s_{m}\right)}\left(\mathbb{R}^{n}\right)}<\infty
$$

for $s_{1}, \ldots, s_{m} \in(n / 2, n]$ and let $T_{\sigma}$ be the operator defined by (1). If $1<p_{j}<\infty, t_{j}=n / s_{j}$ and $0<\delta<\varepsilon<r / m$, where $1 / r=1 / r 1+\ldots+1 / r m, r_{j}=\epsilon_{j} t_{j}$ and $1<\epsilon_{j}<\min \left\{p_{j}, s_{j} /\left(s_{j}-\right.\right.$ $\left.1), 2 s_{j} / n\right\}$, and let $\vec{b} \in B M O^{m}$. Then for any $\vec{\gamma}>\vec{r}$, that is,

$$
\begin{aligned}
& \left|T_{\sigma}\left(f_{1}^{\alpha_{1}}, \ldots, f_{m}^{\alpha_{m}}\right)(z)-T_{\sigma}\left(f_{1}^{\alpha_{1}}, \ldots, f_{m}^{\alpha_{m}}\right)(x)\right| \\
& \quad \leq C \mathscr{M}_{\vec{r}}(\vec{f})(x) .
\end{aligned}
$$

$$
\begin{aligned}
& \leq C \sum_{k=0}^{\infty} 2^{-k\left(s_{1}+\cdots+s_{m}-n / r_{1}-\cdots-n / r_{m}\right)} \mathscr{M}_{\vec{r}}(\vec{f})(x) \\
& \leq C \mathscr{M}_{\vec{r}}(\vec{f})(x) .
\end{aligned}
$$

It remains to consider the case that there exists a proper subset $\left\{j_{1}, \ldots, j_{\gamma}\right\}$ of $\{1, \ldots, m\}, 1 \leq \gamma<m$, such that $\alpha_{j_{1}}=\cdots=$ $\alpha_{j_{\gamma}}=0$. Without loss of generality, we write, for the case $\left\{j_{1}, \ldots, j_{\gamma}\right\}=\{1, \ldots, \gamma\}$, $\gamma_{j}>r_{j}, j=1, \ldots, m$, there exists some constant $C>0$ such that

$$
\begin{aligned}
M_{\delta}^{\sharp} & \left(T_{\sigma, \Sigma \mathbf{b}}(\vec{f})\right)(x) \\
& \leq C\|\vec{b}\|_{B M O^{m}}\left(M_{\varepsilon}\left(T_{\sigma}(\vec{f})\right)(x)+\mathscr{M}_{\vec{\gamma}}(\vec{f})(x)\right),
\end{aligned}
$$

for all $m$-tuples $\vec{f}=\left(f_{1}, \ldots, f_{m}\right)$ of bounded measurable functions with compact support.

Proof. By linearity it is sufficient to consider the particular case when $\vec{b}=b \in B M O$. Fix $b \in \mathrm{BMO}$ and consider the operator

$$
T_{\sigma, b}(\vec{f})(x)=b(x) T_{\sigma}(\vec{f})(x)-T_{\sigma}\left(b f_{1}, f_{2}, \ldots, f_{m}\right)(x) .
$$


Fix $x \in \mathbb{R}^{n}$, for any cube $Q$ with center at $x$; set $\lambda=b_{\mathrm{Q}^{*}}$, where $Q^{\star}=4 \sqrt{n} Q$. We have

$$
\begin{aligned}
& T_{\sigma, b}(\vec{f})(x) \\
& \quad=(b(x)-\lambda) T(\vec{f})(x)-T_{\sigma}\left((b-\lambda) f_{1}, f_{2}, \ldots, f_{m}\right)(x) .
\end{aligned}
$$

Since $0<\delta<r / m<1$,

$$
\begin{aligned}
& \left(\left.\frac{1}{|Q|} \int_{Q}|| T_{\sigma, b}(\vec{f})(z)\right|^{\delta}-|c|^{\delta} \mid d z\right)^{1 / \delta} \\
& \leq\left(\frac{1}{|Q|} \int_{Q}\left|T_{\sigma, b}(\vec{f})(z)-c\right|^{\delta} d z\right)^{1 / \delta} \\
& \leq\left(\frac{C}{|Q|} \int_{Q}\left|(b(z)-\lambda) T_{\sigma}(\vec{f})(z)\right|^{\delta} d z\right)^{1 / \delta} \\
& \quad+\left(\frac{C}{|Q|} \int_{Q}\left|T_{\sigma}\left((b-\lambda) f_{1}, \ldots, f_{m}\right)(z)-c\right|^{\delta} d z\right)^{1 / \delta} \\
& :=A+B .
\end{aligned}
$$

By the John-Nirenberg inequality and Hölder inequality, one has, for $1<q<\epsilon / \delta$ such that $q^{\prime} \delta>1$,

$$
\begin{aligned}
A \leq & C\left(\frac{1}{|Q|} \int_{Q}|b(z)-\lambda|^{q^{\prime} \delta} d z\right)^{1 / q^{\prime} \delta} \\
& \times\left(\frac{1}{|Q|} \int_{Q}\left|T_{\sigma}(\vec{f})(z)\right|^{q \delta} d z\right)^{1 / q \delta} \\
\leq & C\|b\|_{\mathrm{BMO}} M_{q \delta}\left(T_{\sigma}(\vec{f})\right)(x) \\
\leq & C\|b\|_{\mathrm{BMO}} M_{\epsilon}\left(T_{\sigma}(\vec{f})\right)(x) .
\end{aligned}
$$

To estimate term $B$, we split each function $f_{j}$ as $f_{j}=f_{j}^{0}+f_{j}^{\infty}$, where $f_{j}^{0}=f_{j} \chi_{Q^{\star}}$ for $j=1, \ldots, m$. We also have the same decomposition,

$$
\begin{aligned}
& \prod_{j=1}^{m} f_{j}\left(y_{j}\right) \\
& \quad=\prod_{j=1}^{m}\left(f_{j}^{0}\left(y_{j}\right)+f_{j}^{\infty}\left(y_{j}\right)\right) \\
& \quad=\sum_{\alpha_{1}, \ldots, \alpha_{m} \in\{0, \infty\}} f_{1}^{\alpha_{1}}\left(y_{1}\right) \cdots f_{m}^{\alpha_{m}}\left(y_{m}\right) \\
& \quad=\prod_{j=1}^{m} f_{j}^{0}\left(y_{j}\right)+\sum_{\alpha_{1}, \ldots, \alpha_{m} \in \mathcal{F}} f_{1}^{\alpha_{1}}\left(y_{1}\right) \cdots f_{m}^{\alpha_{m}}\left(y_{m}\right),
\end{aligned}
$$

where $\mathscr{I}=\left\{\alpha_{1}, \ldots, \alpha_{m}\right.$ : there is atleast one $\left.\alpha_{j} \neq 0\right\}$.
Taking $c=\sum_{\alpha_{1}, \ldots, \alpha_{m} \in \mathcal{I}} T_{\sigma}\left((b-\lambda) f_{1}^{\alpha_{1}} \cdots f_{m}^{\alpha_{m}}\right)$, we have

$$
\begin{gathered}
B \leq C\left\{\left(\frac{1}{|Q|} \int_{Q}\left|T_{\sigma}\left((b-\lambda) f_{1}^{0}, \ldots, f_{m}^{0}\right)(z)\right|^{\delta} d z\right)^{1 / \delta}\right. \\
+\sum_{\alpha_{1}, \ldots, \alpha_{m} \in \mathcal{F}}\left(\frac{1}{|Q|} \int_{Q} \mid T_{\sigma}\left((b-\lambda) f_{1}^{\alpha_{1}}, \ldots, f_{m}^{\alpha_{m}}\right)(z)\right. \\
-T_{\sigma}\left((b-\lambda) f_{1}^{\alpha_{1}}, \ldots, f_{m}^{\alpha_{m}}\right) \\
\left.\left.\times\left.(x) d z\right|^{\delta}\right)^{1 / \delta}\right\} \\
:=B_{1}+B_{2} .
\end{gathered}
$$

By using Kolmogorov's inequality and Hölder's inequality, one has

$$
\begin{aligned}
B_{1} \leq & C\left\|T_{\sigma}\left((b-\lambda) f_{1}^{0}, \ldots, f_{m}^{0}\right)(z)\right\|_{L^{\gamma, \infty}(\mathrm{Q}, d x /|\mathrm{Q}|)} \\
\leq & C\left(\frac{1}{\left|Q^{\star}\right|} \int_{\mathrm{Q}^{\star}}\left|(b-\lambda) f_{1}^{0}(z)\right|^{r_{1}} d\right)^{1 / r_{1}} \\
& \times \prod_{j=2}^{m}\left(\frac{1}{\left|Q^{\star}\right|} \int_{\mathrm{Q}^{\star}}\left|f_{j}^{0}(z)\right|^{r_{j}} d z\right)^{1 / r_{j}} \\
\leq & C\|b\|_{\mathrm{BMO}} \mathscr{M}_{\vec{\gamma}}(\vec{f}) .
\end{aligned}
$$

By the same argument in the proof of Lemma 14, we have the following estimate:

$$
\begin{aligned}
& \sum_{\alpha_{1}, \ldots, \alpha_{m} \in \mathscr{F}}\left|T_{\sigma}\left(f_{1}^{\alpha_{1}} \cdots f_{m}^{\alpha_{m}}\right)(z)-T_{\sigma}\left(f_{1}^{\alpha_{1}} \cdots f_{m}^{\alpha_{m}}\right)(x)\right| \\
& \leq C \sum_{k=0}^{\infty} 2^{-k\left(s_{1}+\cdots+s_{m}-n / r_{1}-\cdots-n / r_{m}\right)} \\
& \quad \times\left(\frac{1}{\left|2^{k+1} Q^{\star}\right|} \int_{2^{k+1} Q^{\star}}\left|(b-\lambda) f_{1}^{\alpha_{1}}(z)\right|^{r_{1}} d z\right)^{1 / r_{1}} \\
& \quad \times \prod_{j=2}^{m}\left(\frac{1}{\left|2^{k+1} Q^{\star}\right|} \int_{2^{k+1} Q^{\star}}\left|f_{j}^{\alpha_{j}}(z)\right|^{r_{j}} d z\right)^{1 / r_{j}} \\
& \leq C \mathscr{M}_{\vec{r}}(\vec{f})(x) .
\end{aligned}
$$

This completes the proof. 


\section{Proof of Theorems}

Proof of Theorem 2. By Lemmas 8 and 14, we have

$$
\begin{aligned}
& \left\|T_{\sigma}(\vec{f})\right\|_{L^{p, \kappa}\left(v_{\vec{\omega}}\right)} \\
& \quad \leq\left\|M_{\delta}\left(T_{\sigma}(\vec{f})\right)\right\|_{L^{p, \kappa}\left(v_{\vec{\omega}}\right)} \\
& \leq C\left\|M_{\delta}^{\sharp}\left(T_{\sigma}(\vec{f})\right)\right\|_{L^{p, \kappa}\left(v_{\vec{\omega}}\right)} \\
& \leq C\left\|\mathscr{M}_{\vec{r}}(\vec{f})\right\|_{L^{p, \kappa}\left(v_{\vec{\omega}}\right)} \\
& \leq C \prod_{j=1}^{m}\left\|f_{j}\right\|_{L^{p j, \kappa}} .
\end{aligned}
$$

Proof of Theorem 3. By Lemma 13, there are $\epsilon_{j}^{\prime}<p_{j} / r_{j}$ such that $\omega_{j} \in A_{p_{j} /\left(r_{j} \epsilon_{j}^{\prime}\right)}$. Let $\gamma_{j}=r_{j} \epsilon_{j}^{\prime}$; by Lemmas 8 and 15 , one has

$$
\left\|\mathscr{M}_{\vec{\gamma}}(\vec{f})\right\|_{L^{p, \kappa}\left(\nu_{\vec{\omega}}\right)} \leq C \prod_{j=1}^{m}\left\|f_{j}\right\|_{L^{p_{j}, \kappa}}\left(\omega_{j}\right),
$$

and then

$$
\left\|T_{\sigma, \Sigma \mathbf{b}}(\vec{f})\right\|_{L^{p, \kappa}\left(v_{\vec{\omega}}\right)} \leq C \prod_{j=1}^{m}\left\|f_{j}\right\|_{L^{p_{j}, \kappa}\left(\omega_{j}\right)} .
$$

[6] M. Fujita and N. Tomita, "Weighted norm inequalities for multilinear Fourier multipliers," Transactions of the American Mathematical Society, vol. 364, no. 12, pp. 6335-6353, 2012.

[7] K. Li, Q. Xue, and K. Yabuta, "Weighted version of Carleson measure and multilinear Fourier multiplier," Forum Mathematicum, 2012.

[8] G. Hu and Q. Lin, "Weightednorm inequalities for multilinear singular integral operators and applications," http://arxiv. org/abs/1208.6346.

[9] T. A. Bui and X. T. Duong, "Weighted norm inequalities for multilinear operators and applications to multilinear Fourier multipliers," Bulletin des Sciences Mathématiques, vol. 137, no. 1, pp. 63-75, 2013.

[10] K. Li and W. Sun, "Weighted estimates for multilinear Fourier multiplier," Forum Mathematicum, 2013.

[11] Y. Jiao, "A weighted norm inequalities for multilinear Fourier multipliers operators," Mathematical Inequalities and Applications, preprint.

[12] A. K. Lerner, S. Ombrosi, C. Pérez, R. H. Torres, and R. TrujilloGonzález, "New maximal functions and multiple weights for the multilinear Calderón-Zygmund theory," Advances in Mathematics, vol. 220, no. 4, pp. 1222-1264, 2009.

[13] Y. Komori and S. Shirai, "Weighted Morrey spaces and a singular integral operator," Mathematische Nachrichten, vol. 282, no. 2, pp. 219-231, 2009.

[14] L. Hörmander, "Estimates for translation invariant operators in $L^{p}$ spaces," Acta Mathematica, vol. 104, pp. 93-140, 1960.

[15] C. Fefferman and E. M. Stein, " $H^{p}$ spaces of several variables," Acta Mathematica, vol. 129, no. 3-4, pp. 137-193, 1972.

\section{Conflict of Interests}

The authors declare that they have no conflict of interests.

\section{Acknowledgments}

The authors would like to thank the referee for some very valuable suggestions. This research was supported by the NSF of China (no. 11161044 and no. 11261055) and by XJUBSCX2012004.

\section{References}

[1] R. R. Coifman and Y. Meyer, "Au delà des opérateurs pseudodifférentiels," Astérisque, vol. 57, pp. 1-185, 1978.

[2] L. Grafakos and R. H. Torres, "Multilinear Calderón-Zygmund theory," Advances in Mathematics, vol. 165, no. 1, pp. 124-164, 2002.

[3] N. Tomita, "A Hörmander type multiplier theorem for multilinear operators," Journal of Functional Analysis, vol. 259, no. 8, pp. 2028-2044, 2010.

[4] L. Grafakos and Z. Si, "The Hörmander multiplier theorem for multilinear operators," Journal für die Reine und Angewandte Mathematik, vol. 668, pp. 133-147, 2012.

[5] A. Miyachi and N. Tomita, "Minimal smoothness conditions for bilinear Fourier multipliers," Revista Matemática Iberoamericana, vol. 29, no. 2, pp. 495-530, 2013. 


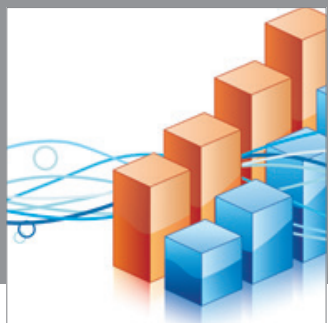

Advances in

Operations Research

mansans

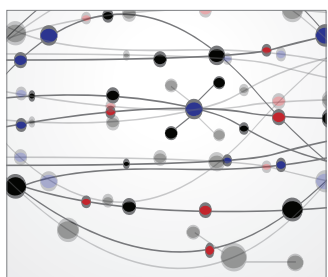

The Scientific World Journal
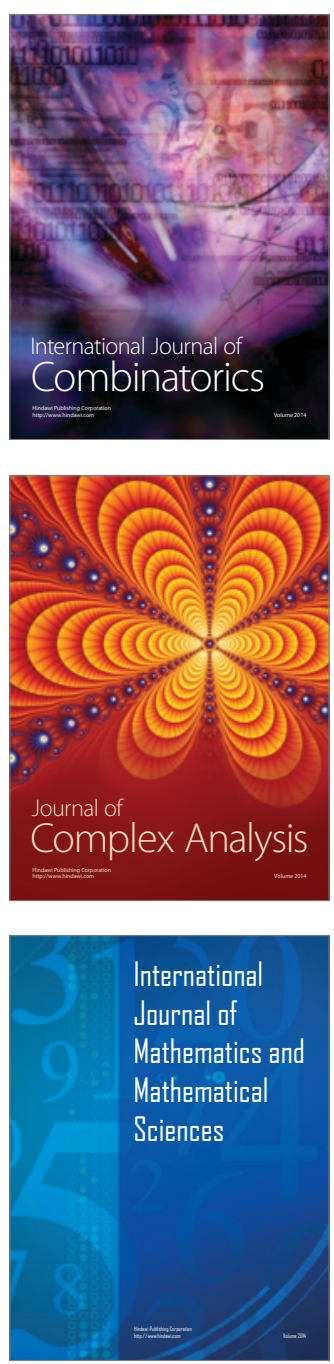
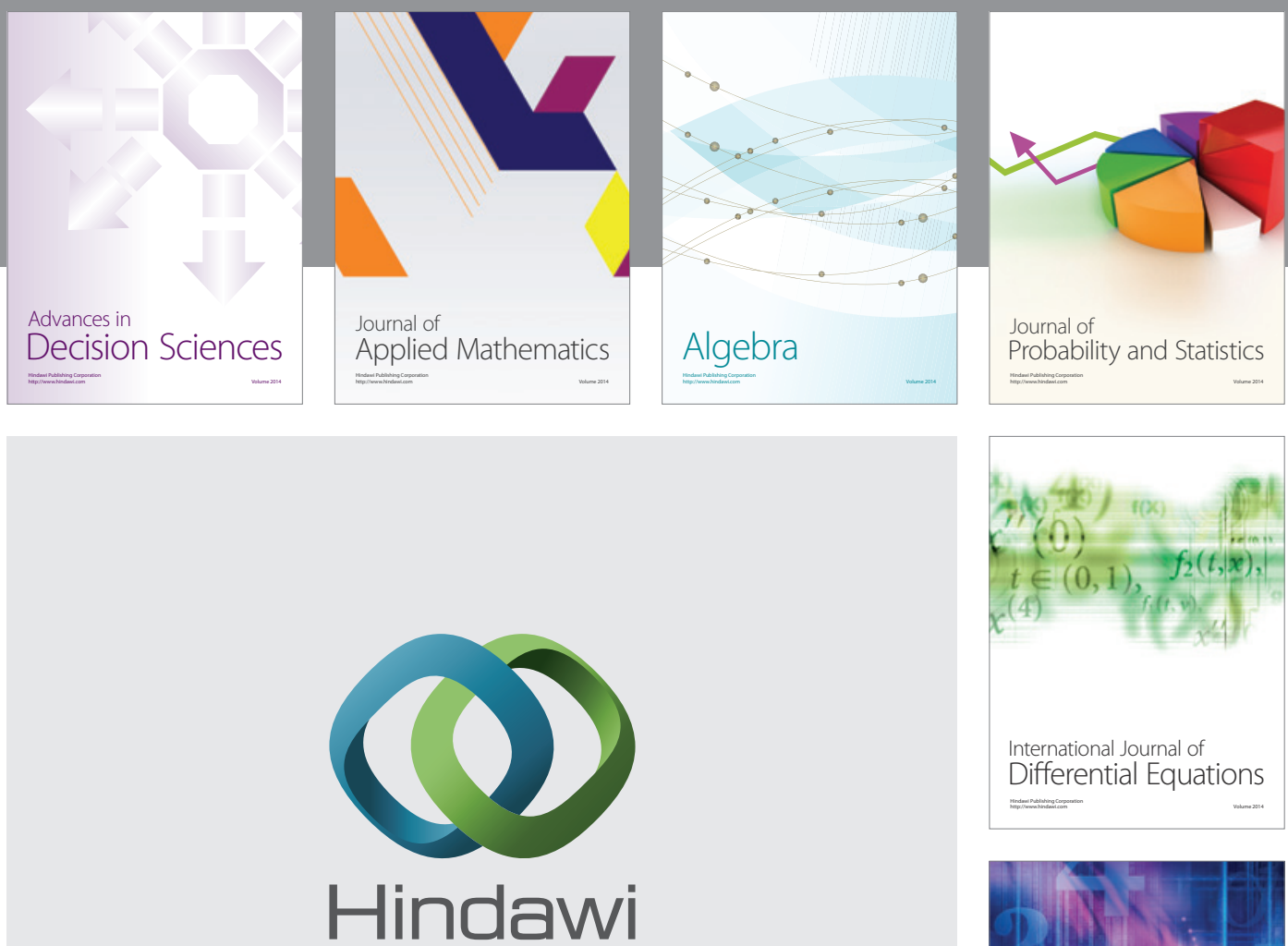

Submit your manuscripts at http://www.hindawi.com
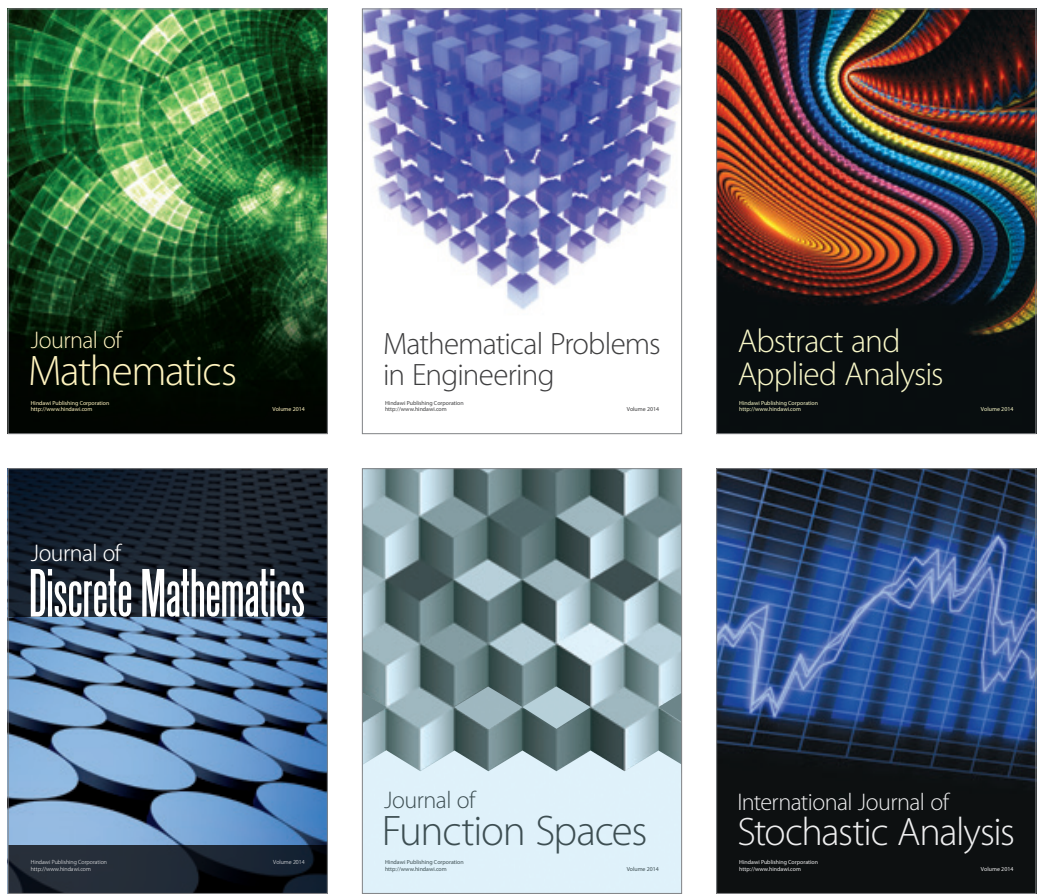

Journal of

Function Spaces

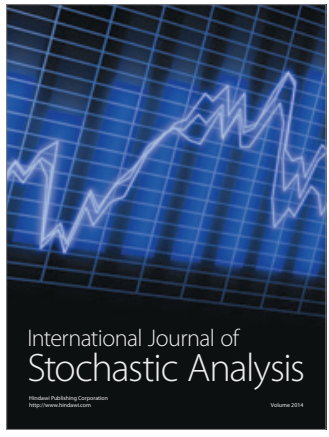

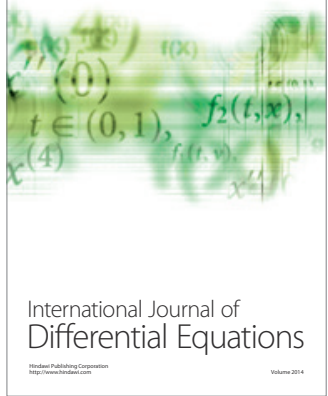
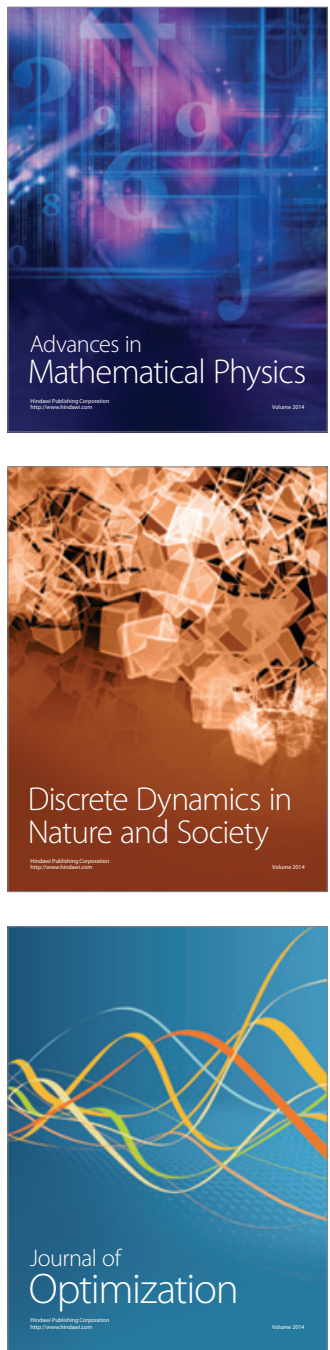